\title{
Absolute and Geometric Parameters of Contact Binary GW Cnc
}

\author{
B. Gürol ${ }^{\mathrm{a}}$, G. Gökay ${ }^{\mathrm{a}}$, G. Saral ${ }^{\mathrm{b}, \mathrm{c}}$, S.H. Gürsoytrak ${ }^{\mathrm{a}}$, S. Cerit ${ }^{\mathrm{a}}$, Z. Terzioğlu ${ }^{\mathrm{a}}$ \\ ${ }^{a}$ Ankara University, Science Faculty, Dept. of Astronomy and Space Sciences, 06100 \\ Tandoğan, Ankara, Turkey \\ ${ }^{b}$ Istanbul University, Graduate School of Science and Engineering, Bozdoğan Kemeri \\ Cad. 8, Vezneciler, Istanbul, Turkey \\ ${ }^{c}$ Harvard-Smithsonian Center for Astrophysics, 60 Garden Street MS 66, Cambridge MA \\ 02138, USA
}

\begin{abstract}
We present the results of our investigation on the geometrical and physical parameters of the W UMa type binary system GW Cnc. We analyzed the the photometric data obtained in 2010 and 2011 at Ankara University Observatory (AUO) and the spectroscopic data obtained in 2010 at TUBITAK National Observatory (TUG) by using the Wilson-Devinney (2013 revision) code to obtain the absolute and geometrical parameters. We derived masses and radii of the eclipsing system to be $M_{1}=0.257 M_{\odot}$, $M_{2}=0.971 M_{\odot}, R_{1}=0.526 R_{\odot}$ and $R_{2}=0.961 R_{\odot}$ with an orbital inclination $i\left(^{\circ}\right)=83.38 \pm 0.25$ and we determined the GW Cnc system to be a W-type W UMa over-contact binary with a mass ratio of $q=3.773 \pm 0.007$.
\end{abstract}

Keywords:

Stars: binaries: eclipsing, Stars: fundamental parameters, Technique: photometry \& spectroscopy, Stars: individual (GW Cnc)

\section{Introduction}

The short-period $\left(P \sim 0^{d} .2814\right)$ binary system GW Cnc (ASAS 084813+2107.2, $\left.V=12^{m} .595, \alpha_{2000}=08^{h} 48^{m} 12^{s} .693, \delta_{2000}=+21^{\circ} 07^{\prime} 13^{\prime \prime} .86\right)$ was reported as an $L$ : type variable by Takamizawa (2000) in the GCVS. Thereafter,

Email address: Birol.Gurol@ankara.edu.tr (B. Gürol)

Preprint submitted to New Astronomy

December 3, 2015

(C) 2015. This manuscript version is made available under the Elsevier user license

http://www.elsevier.com/open-access/userlicense/1.0/ 
Khruslov (2005) identified the system as W UMa (EW) type eclipsing binary by using ASAS3 and ROTSE data and gave the light elements as follows:

$$
H J D(\text { Min.I })=2451554.023+0^{d} .281415 \times E .
$$

The first photometric analysis of the system was made by Terrell et al. (2005) which gives the mass ratio of the system as 4.15 and the orbital inclination as $83^{\circ} .4$. The $(\mathrm{B}-\mathrm{V})$ color was obtained by the same authors as $0^{m} .68$ during the total eclipse and also at the phase of 0.75 . The spectral type of the system and distance are given as $G 8 \mathrm{~V}$ and $319 \mathrm{pc}$, respectively in the catalogue of "All-sky spectrally-matched Tycho2 stars" (Pickles \& Depagne , 2010).

In this study, we derive the physical and geometrical parameters of the eclipsing binary system using photometric data obtained at the Ankara University Observatory (AUO) between 2010 October and 2011 March and radial velocity data obtained at the TUBITAK National Observatory (TUG) in 2010 November. We revised the light elements by using the published minima times given in the literature within the framework of the well-known (O-C) method.

\section{Observations}

Observations of GW Cnc in $V R I$ bands were carried out with an Apogee ALTA-U47 (1024x1024) CCD photometric system attached to the $0.40 \mathrm{~m}$ Meade-LX200GPS telescope at the AUO in 2010 and 2011. The telescope was equipped with a standard Johnson $U B V R I$ filter set with a field of view of $11^{\prime} .3 \times 11^{\prime} .3$. The exposure time for each image was varied according to the filter used.

TYC1399-1572-1 $\left(V=11^{m} .43, \alpha_{2000}=08^{h} 48^{m} 38^{s} .353, \delta_{2000}=+21^{\circ} 05^{\prime} 10^{\prime \prime} .68\right)$, GSC1399-1348 $\left(V=12^{m} .96, \alpha_{2000}=08^{h} 48^{m} 22^{s} .10, \delta_{2000}=+20^{\circ} 59^{\prime} 58^{\prime \prime} .00\right)$ and GSC1399-2073 $\left(V=12^{m} .84, \alpha_{2000}=08^{h} 48^{m} 29^{s} .70, \delta_{2000}=+21^{\circ} 00^{\prime} 36^{\prime \prime} .7\right)$ were used as comparison and check stars which are located on the same frame of the CCD camera as GW Cnc. The coordinates were obtained from SIMBAD database. We give the information about the observations in Table 1 showing the date, the number of observations, nightly standard deviation of observations and observers. In total, 2493 filtered observations were obtained between the years 2010 and 2011.

Table 1. 
The photometric calibration of the raw CCD images was performed according to the standard method using the program package C-Munipack (MuniWin, Version 2.0.10) programmed by Motl (2012). All CCD images were reduced by means of the "Advanced Calibration Scheme" using bias, scalable dark and flat files. The standard errors of the observations were estimated by using the difference between the magnitude of the check and comparison star which are constant within $\pm 0^{m} .015, \pm 0^{m} .014$ and $\pm 0^{m} .016$ for $V, R$ and $I$ bands, respectively for all of the observations. Differential extinction corrections were found to be negligible, since the comparison stars are very close to the variable star. The data may be obtained from authors upon request.

The magnitude and color values for variable and comparison stars are listed in Table 2. The values were obtained from the "All-sky spectrally matched Tycho2 stars" catalogue which were calculated by fitting $U B V R I-$ $Z Y$ and $u^{\prime} g^{\prime} r^{\prime} i^{\prime} z^{\prime}$ magnitudes, spectral types, and distances for 2.4 million stars, derived from synthetic photometry of a library spectrum that best matches the Tycho2 $B_{T}, V_{T}$, NOMAD RN, and 2MASS JHK2/S catalogue magnitudes (Pickles \& Depagne, 2010).

Table 2 .

\section{Light Elements}

We determined new light elements for the eclipsing system since the orbital period of the system and period variations are important for the remaining analysis. First, we collected a total of 21 timings of minimum light given in Table 3 and calculated the $\mathrm{O}-\mathrm{C}$ variation as shown in Fig. 1 by using the light elements given by Khruslov (2005).

Table 3 .

Figure 1.

Essentially, we used a total of 19 minima times to revise the ephemeris given by the author. Two timings of minima light are excluded from the fit and marked in the Fig.1. Because of the limited minima times and small time spans, we were able to do only a linear fit on the O-C variation to obtain the corrections on the epoch and period of the eclipsing system. The derived 
coefficients of the fit are $(O-C)=-3.602352539 \cdot 10^{-6} \times E+0.02094661412$. Using these fit parameters, we revised the ephemeris as,

$$
H J D(M i n . I)=2451554.0439(18)+0^{d} 2814114(1) \times E,
$$

where the uncertainties are given in parenthesis. The improved ephemeris was used for the rest of the calculations in this study.

\section{Light Curves}

In Fig. 2 we plotted all of the observations obtained in 2010 and 2011 with respect to the orbital phase. Because of the nightly observational error on the differential magnitude (Table 1) is nearly same with the scatter of the observations we prefer averaging the observed magnitudes at 0.01 phase intervals. In this way we can obtain most accurate light curve that represents the system and also the computational time decreases depending on fewer and more accurate observational data.

As can be seen from the $V R I$ light curves given in Fig.2 the primary minima are complete or on the limit of complete eclipses. We can say that the luminosities of the components are nearly the same since the primary and secondary minima do not vary in depth. On the other hand, some W UMa type systems may show two different maxima which is called the O'Connell effect (O'Connell, 1951). On the $B, V$ and $I_{c}$ band light curves of $\mathrm{GW}$ Cnc published by Terrell et al. (2005) there was no prominent O'Connell effect, however; we see the O'Connell effect on the primary maxima which are $\sim 0^{m} .02$ lower than the secondary maxima, which can be a sign of a changing activity on the component's surface. For systems in which one or both components are F to K stars, we can expect solar like activity such as photospheric spots, chromospheric emission, coronal X-ray, radio emission and flare activity (Kang et al. , 2002).

Figure 2.

\section{Radial Velocity and Spectral Type}

Spectroscopic observations were obtained with the Turkish Faint Object Spectrograph Camera (TFOSC) attached to the $1.50 \mathrm{~m}$ telescope on 2010 November 20 and 21. Further details on the telescope and the spectrograph can be found at http: //www.tug.tubitak.gov.tr. The wavelength coverage 
of each spectrum is from 3850 to $9120 \AA$ in 11 orders, with a resolving power of 7000 at $6563 \AA$. We also obtained a high S/N spectrum of the HD193664 $\left(v_{r}=-4.7 \mathrm{~km} / \mathrm{s}, \mathrm{G} 3 \mathrm{~V}\right)$ to use as a template to derive the radial velocities of the binary.

We took a total of 11 spectra using an exposure time of $900 \mathrm{~s}$. Bias subtraction and flat-field correction were made with standard IRAF ${ }^{1}$ procedures. The spectra were extracted using procedures from the IRAF package noao.imred.echelle. Wavelength calibration was performed using a Fe-Ar comparison lamp during the same observing run. $\Delta \lambda$ values are different for the 11 orders and changing between 0.288 and $0.751 \AA / p x$. The velocity dispersion is changing between $22-27 \mathrm{~km} / \mathrm{s} / \mathrm{px}$ for different orders.

The exposure times given for the observations of GW Cnc are nearly $3.7 \%$ of the orbital period and this is slightly longer than the recommended values given by Hilditch (2001) ( $1-2 \%$ of the orbital phase). The heliocentric correction was applied with the RVCORR and DOPCOR tasks of the IRAF packages ASTUTIL and ECHELLE. Radial velocities were determined by using the IRAF package RV (CCF) for suitable orders. All of the velocities obtained from suitable orders were averaged and used to calculate the standard errors on the velocities. The derived radial velocities of the components are given in Table 4.

Table 4.

According to the radial velocity curves, we confirm the system to be a Wtype W UMa system, i.e. the larger, more massive component has the lower surface temperature but contributes more overall brightness to the eclipsing system. This larger, cooler and more massive star is the one which is eclipsed during the secondary minima. We obtained the spectral type of the system by comparing the spectral observation near secondary minima to the spectral standards found in the literature and using the cross correlation function (CCF) in IRAF. In Fig. 3 we give the variation of HGHT parameter with respect to the coded spectral types $(\mathrm{O}=0, \mathrm{~B}=1, \mathrm{~A}=2, \ldots)$. The comparison is getting higher (HGHT)-values when two spectra are matching with each other. This method is explained in the document published by Skinas

\footnotetext{
${ }^{1} \mathrm{IRAF}$ is distributed by the National Optical Astronomical Observatories, operated by the Association of the Universities for Research in Astronomy, Inc., under cooperative agreement with the National Science Foundation.
} 
Observatory with the Project number 8: Stellar Spectra Classification ${ }^{2}$. By using this method we determined the spectral type of the hotter component as K0V. However, there is another peak at the hotter side as seen in Fig. 3 which is corresponding to a G2IV type star. The analyses were made for apertures 5 and 6 and the final determination corresponds to a spectral type of K0V or G2IV with the corresponding temperatures $T_{\text {eff }}=5240 \mathrm{~K}$ or 5790 K, respectively according to Allen's Astrophysical Quantities (Cox , 2000).

Figure 3.

The distance of the system was given as 319 pc by Pickles \& Depagne (2010), and according to this distance, the magnitudes/colors of the system must be affected by the interstellar medium. The Period-Color relation given by Wang (1994), under the assumption that the components in a contact binary system are formed from almost normal hydrogen-core-burning stars that obey the mass-radius relation for main sequence stars, predicts that the intrinsic color index can be written as:

$$
(B-V)_{0}=0.077-1.003 \log P(\text { day })
$$

where $P$ is the orbital period in days. For this equation, the correlation coefficient to the fit is given as 0.78 by Wang (1994). This indicates that there is considerable scatter to the fit when compared to the actual observed data points. Using the period obtained in our study we derived the $(B-$ $V)_{0}$ intrinsic color index for the eclipsing system as $0^{m} .629$. The difference between this value and the color given by Terrell et al. (2005) is $0^{m} .051$. The corresponding temperature and spectral type are $5790 \mathrm{~K}$ and G2 (Cox , 2000), respectively which is nearly $550 \mathrm{~K}$ hotter than the K0V type stars but it is the same as for G2IV type stars. In all our solutions, we set the temperature of the hotter component as $T_{1}=5790 \mathrm{~K}$ based on the PeriodColor relation and on our derived spectral type for this system.

\section{Figure 4.}

We obtained the differential colors of the system (Fig. 4) with respect to the orbital phase since we did not obtain the magnitudes in the standard system. It can be seen that the colors do not vary more than $\sim 0^{m} .02$ at

\footnotetext{
${ }^{2}$ http://skinakas.physics.uoc.gr/en/
} 
the primary and secondary minima. Terrell et al. (2005) did not find any change on the $(\mathrm{B}-\mathrm{V})$ color for different phases, either.

\section{Light Curve Analysis}

The Wilson-Devinney method (Wilson \& Devinney , 1971; Wilson , 1978, 1979, 1990; Wilson \& van Hamme , 2007) was applied to solve the system parameters of GW Cnc. We used the 2013 revision of the program which operates under the interface of MS Excel Software, programmed by B.Gürol for Windows. The method computes the light curves as a function of the following parameters: the orbital inclination $i$, surface potentials $\Omega_{1,2}$, fluxweighted average surface temperatures $T_{1,2}$, mass ratio $q=m_{2} / m_{1}$, normalized monochromatic luminosities $L_{1,2}$, limb-darkening coefficients $x_{1,2}$, gravity darkening exponents $g_{1,2}$, and bolometric albedos $A_{1,2}$ by assuming the stellar surfaces to be equipotentials. The subscripts 1 and 2 refer to the primary (hotter, less massive) and secondary (cooler, more massive) components, respectively.

The linear and logarithmic limb darkening coefficients for bolometric calculations were taken from van Hamme (1993). The gravity darkening exponents and the bolometric albedos were set to be 0.32 (Lucy , 1967) and 0.50 (Ruciński , 1969), respectively for both components, according to the convective atmospheres. The grid resolution values were taken as 30, 30, 15, 15 for N1, N2, N1L and N2L, respectively by using the detailed reflection model of Wilson (1990) $\mathrm{MREF}=2$ and number of reflections with $\mathrm{NREF}=2$. In the Wilson \& Devinney code N1 and N2 are used for grid sizes and N1L and N2L for coarse grid integers, respectively for star 1 and 2. A circular orbit and synchronous rotation were assumed as expected for overcontact binary systems.

For our solutions we assumed that the system is in contact, employing the Mode 3 option of the computing code (overcontact mode). The adjustable parameters for the simultaneous fitting of the light curves were: the inclination $i$, the mean surface temperature of secondary component $T_{2}$, the nondimensional surface potential of primary component $\Omega_{1}$ and the monochromatic luminosity of the primary component $L_{1}$. Simultaneously, the radial velocity parameters; the semi-major axis $a$, velocity of the center of the mass $V_{\gamma}$ and the mass ratio of the system $q=m_{2} / m_{1}$ were adjusted. We selected $\mathrm{IPB}=0$, thus the temperature of the secondary component was used to calculate the $L_{2}$ values. 
The photometric mass ratio and orbital inclination were determined by (Terrell et al. , 2005) as $q=4.15 \pm 0.01$ and $i=83^{\circ} .4 \pm 0.4$, respectively. With the initial parameters, we solved the light and radial velocity curves simultaneously until the solution was converged. The convergent solution was obtained with the adjustable parameters by iterating, until the correction on the parameters became smaller than the corresponding standard deviations. After that we placed a dark spot on the secondary component (prefered) to account of the O'Connell effect seen on the secondary maxima and obtained better solution by including the parameters of Latitude, Longitude, Radius and Temperature Factor of the spot. The theoretical light and radial velocity curves are calculated with the final elements and shown in Figures 5 and 6 . The results of the final solution are given in Table 5 and the configuration of GW Cnc calculated with the Roche model is shown in Figure 7.

Figure 5.

Figure 6.

Table 5.

Figure 7 .

\section{Absolute Parameters}

Combining the parameters of the photometric and spectroscopic orbital solutions, we derived absolute parameters for the components. Using the orbital period $P=0^{d} .2814114$, and semi-major axis $a=(1.936 \pm 0.010) \mathrm{R}_{\odot}$ we can calculate the total mass of the system by using Newton's formulation of Kepler's third law ( $P$ in year and $a$ in AU):

$$
a^{3} / P^{2}=\left(M_{1}+M_{2}\right)=(1.228 \pm 0.019) \mathrm{M}_{\odot}
$$

According to the light and radial velocity curve solution, we derived the mass ratio of the system as $q=m_{2} / m_{1}=3.773 \pm 0.007$ and the mass of the primary and the secondary components as $M_{1}=(0.257 \pm 0.004) \mathrm{M}_{\odot}$ and $M_{2}=(0.971 \pm 0.016) \mathrm{M}_{\odot}$, respectively.

The volume radii of the components were estimated with the following formula given by Eggleton (1983), 
and calculated as $r_{1}=0.27186 \pm 0.00016$ and $r_{2}=0.49628 \pm 0.00014$ for primary and secondary components, respectively. By using the values of the semi-major axis and the volume radius, we can find the radii of the components in solar units separately as follows $R_{1}=a \times r_{1}=(0.526 \pm$ $0.003) \mathrm{R}_{\odot}$ and $R_{2}=a \times r_{2}=(0.961 \pm 0.007) \mathrm{R}_{\odot}$. Since the sum of the volume radius of the components is $r_{v o l}=r_{1 v o l}+r_{2 v o l}=0.768>0.75$ the system is in marginal contact (Kopal, 1959).

The absolute parameters $M_{b o l 1,2}$ and $L_{1,2}$ can be calculated using the well-known equations (6) and (7) respectively,

$$
\begin{gathered}
M_{b o l 1,2}=4^{m} .75-5 \log \left(R_{1,2} / \mathrm{R}_{\odot}\right)-10 \log \left(T_{1,2} / \mathrm{T}_{\odot}\right), \\
L_{1,2}=\left(R_{1,2} / \mathrm{R}_{\odot}\right)^{2}\left(T_{1,2} / \mathrm{T}_{\odot}\right)^{4} .
\end{gathered}
$$

Adopting $T_{\odot}=5780 \mathrm{~K}$, the bolometric magnitudes were found as $M_{b o l 1}=$ $6.136 \pm 0.032$ and $M_{b o l 2}=4.936 \pm 0.017$, and luminosities $L_{1}=(0.279 \pm$ $0.008) \mathrm{L}_{\odot}$ and $L_{2}=(0.842 \pm 0.019) \mathrm{L}_{\odot}$ for the primary and secondary component, respectively. The mean densities of the components were derived as $\rho_{1}=2.489 \pm 0.004 \mathrm{gr} / \mathrm{cm}^{3}$ and $\rho_{2}=1.543 \pm 0.002 \mathrm{gr} / \mathrm{cm}^{3}$ by using the equations given below by Mochnacki (1981):

$$
\rho_{1}=\frac{0.0189}{r_{1 \text { mean }}^{3} P^{2}(1+q)} \text { and } \rho_{2}=\frac{0.0189 q}{r_{2 \text { mean }}^{3} P^{2}(1+q)} .
$$

The spectral type of the hotter and cooler components were found to be as $G 2$ and $G 4$ from the tables given by Cox (2000).

Because of the total eclipse at the primary minima, we can obtain the magnitude of the secondary component from the light curve, $V_{\text {sec. }}=13^{m} .020 \pm$ 0.006. We found the Bolometric Correction (BC) as -0.200 and -0.207 for the G2V and G4V spectral types, respectively by using the table of the MK spectral types given in Allen's Astrophysical Quantities (Cox , 2000). Since the bolometric magnitude of the secondary component is known (given in Table 6), we can find the absolute magnitude of the component by using the formula; $M_{2 V}=M_{b o l 2}-B C=5^{m} .143$. The temperature of the secondary component is also known, so we can obtain the intrinsic color $(B-V)_{0}=0.663$ from the tables of Cox (2000). Using this value and the observed color of 
$(B-V)=0.680 \pm 0.02$ given by Terrell et al. (2005), we obtain the Color Excess as $E(B-V)=(B-V)-(B-V)_{0}=0.017 \pm 0.023$, and find the interstellar extinction in the $V$ filter as $A_{V}=3.1 \times E(B-V)=0.053 \pm 0.072$. With those values, we calculated the distance of the secondary component (and thus the distance of the system) as,

$$
d(p c)=367 \pm 2 .
$$

The calculated distance to the eclipsing system is close to the value which was given as $d=319$ pc by Pickles \& Depagne (2010). The V magnitude of the system used in this calculation is $12^{m} .716$ as given in Table 2 and the absolute parameters obtained for GW Cnc are given in Table 6 .

Table 6.

The location of the components of GW Cnc in the $\log T-\log L$ diagram is given in Figure 8, which includes other A- and W-type W UMa systems, obtained from the work of Yakut \& Eggleton (2005). The sense of primary and secondary for the systems plotted in Figure 8 is the reverse of the definition we have selected in our analysis, so we have plotted them in the same sense of Yakut \& Eggleton (2005), i.e., the primary star is the more massive star and the secondary is the less massive star. As can be seen in Figure 8, the primary and secondary components of GW Cnc are located among the W-type systems.

Figure 8.

\section{Results and Discussions}

We derived a combined photometric and spectroscopic solution for the eclipsing binary GW Cnc. It is found that the binary is a W-type over-contact system with a mass ratio of $q=(3.7729 \pm 0.0072)$. The primary eclipse is total and the system has an orbital inclination of $i\left(^{\circ}\right)=(83.380 \pm 0.253)$. The temperature difference between the components is $\Delta T=141 \mathrm{~K}$ and the individual values correspond to spectral types are approximately $\mathrm{G} 2 \mathrm{~V}$ and G4V for the primary and secondary component. The degree of geometrical contact, defined by the fill-out parameter, $f=\left(\Omega_{\text {in }}-\Omega\right) /\left(\Omega_{\text {in }}-\Omega_{\text {out }}\right)$ is $9.41 \%$, which means that the system is an over-contact binary, and is consistent with the W-type W UMa systems (Dryomova et al. , 2006). 


\section{Acknowledgments}

We thank TUBITAK National Observatory (TUG) for a partial support for using the RTT150 telescope with project number 10BRTT150-031 to the observers of the system mentioned in Table 1 . This research has made use of the SIMBAD database, operated at CDS, Strasbourg, France, and NASA's Astrophysics Data System Abstract Service. This publication makes use of data products from the Two Micron All Sky Survey, which is a joint project of the University of Massachusetts and the Infrared Processing and Analysis Center/California Institute of Technology, funded by the National Aeronautics and Space Administration and the National Science Foundation.

\section{References}

Brat, L., Smelcer, L., Kueakova, H., Ehrenberger, R. et al.: 2008, OEJV, 94, 1

Cox, A.N. (Ed.), 2000. Allen's Astrophysical Quantities, fourth ed. SpringerVerlag.

Diethelm, R., 2009, IBVS 5871

Diethelm, R., 2011, IBVS 5992

Diethelm, R., 2012, IBVS 6011

Diethelm, R., 2012, IBVS 6029

Dryomova, G.N. \& Svechnikov, M.A., 2006, Astrophysics, Volume 49, Issue 3, pp.358-369.

Eggleton, P.P., 1983, ApJ 268, 368

Gökay G., Demircan Y., Gürsoytrak, H., Terzioğlu Z., Okan, A., Doğruel, M.B., Saral, G., Cerit, S., Şemuni, M., Kılıç, Y., Çoker D., Derman E. and Gürol B., 2012, IAU Inform. Bull. Var. Stars, No 6039.

Girardi, L., Bressan, A., Bertelli, G., Chiosi, C., 2000. A \& AS 141, 371.

Hilditch, R.W., 2001, An Introduction to Close Binary Stars. Cambridge University Press. ISBN 0-521-24106-5. 
Honkov K., Juryek J., Lehky M., \& 67 co-authors, 2013, B.R.N.O. Contributions No.38 http://var.astro.cz/oejv/issues/oejv0160.pdf

Hübscher, J., 2007, IBVS 5802

Hübscher, J., Lehmann, P.B., Monninger, G., Steinbach, H.-M., Walter, F.: 2010, IBVS 5918

Honkova K., Jurysek J., Lehky M. (+77 author)., 2013, B.R.N.O. Contributions 38

Kang, Y.W., Oh, K.-D., Kim, C.-H., Hwang, C., Kim, Ho-il, Lee, W.-B., 2002, Mon. Not. R. Astron. Soc. 331, 707

Kazuo N.: 2010, Variable Star Bulletin No. 50

Khruslov, A.V., 2005, IBVS 5599

Kopal, Z., 1959, Close Binary Systems, 1959cbs..book.....K.

Lucy, L.B., 1967, Z. Astrophysics, 65, 89.

Mochnacki, S.W., 1981, ApJ 245, 650.

Motl, D., 2012, C-Munipack Project v2.0, http://sourceforge.net/projects/cmunipack/files/

Nelson, Robert H., 2014, IBVS 6092.

O'Connell, D.J.K., 1951, Pub. Riverview College Obs., 2, 85

Pickles, A., and Depagne, E., 2010, Publ. Astron. Soc. Pac. 122, 1437-1464.

Ruciński, S.M. 1969, Acta Astron, 19, 245.

Takamizawa, K., 2000, VSOLJ Variable Star Bulletin 31

Terrell, D., Gross, J., Cooney, W.R, 2005, IBVS 5625

van Hamme, W., 1993, AJ 106, 2096

Wang, J.M., 1994, ApJ 434, 277.

Wilson R.E. \& Devinney, E.J., 1971. ApJ 166, 605. 
Wilson, R.E., 1978, ApJ, 224, 885.

Wilson, R.E., 1979, ApJ, 234, 1054.

Wilson, R.E., 1990, ApJ 356, 613.

Wilson, R.E. \& van Hamme, W., 2007, Documentation of Eclipsing Binary Compute Model. Astronomy Department, University of Florida, Gainesville.

Yakut, K. \& Eggleton, P.P., 2005, ApJ, 629, 1055. 


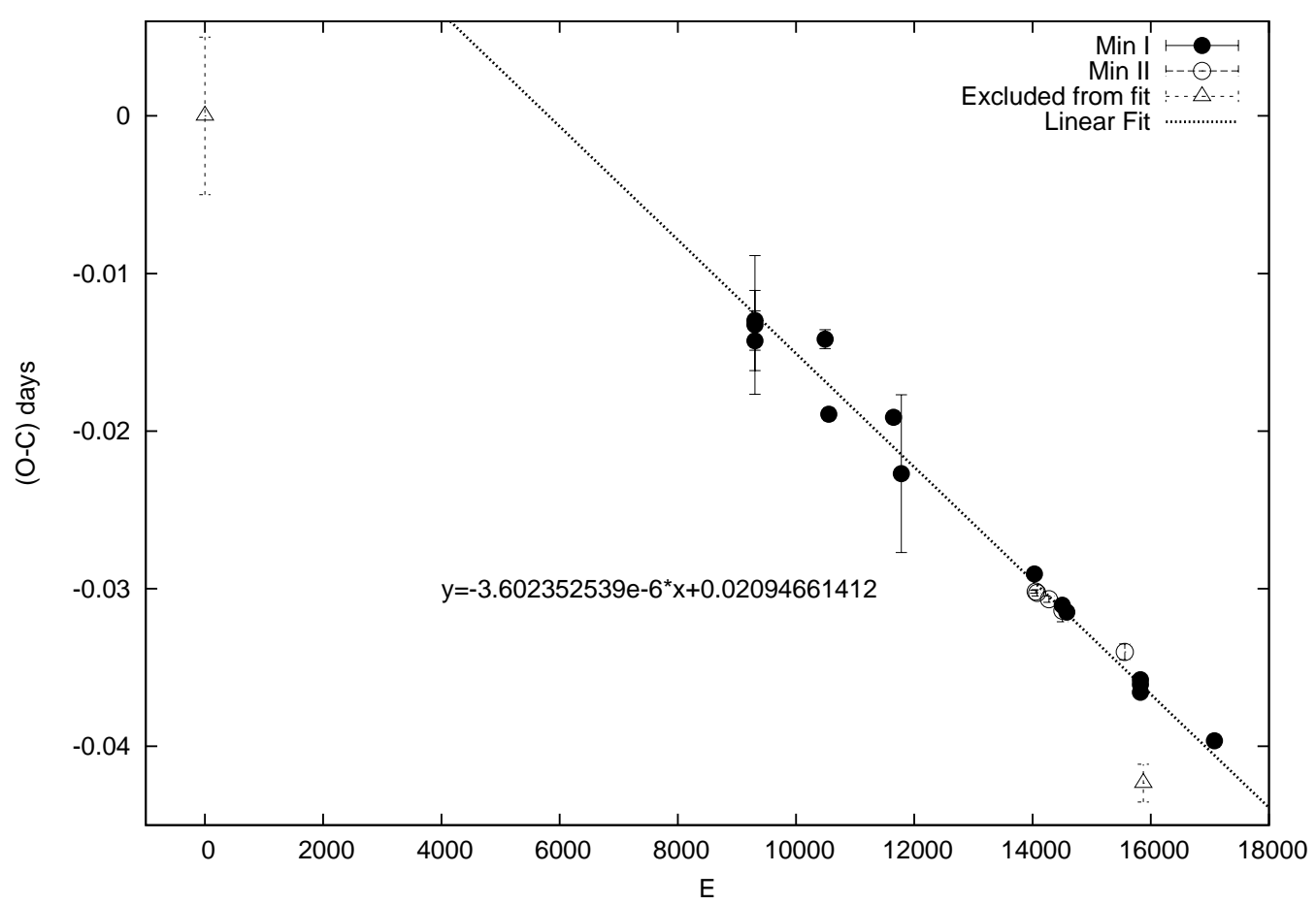

Figure 1: (O-C) variation of GW Cnc. Linear fit of the data by using light elements given by Khruslov (2005). Because the errors of two times of minimum light were not published, we assigned estimated mean errors for them. 


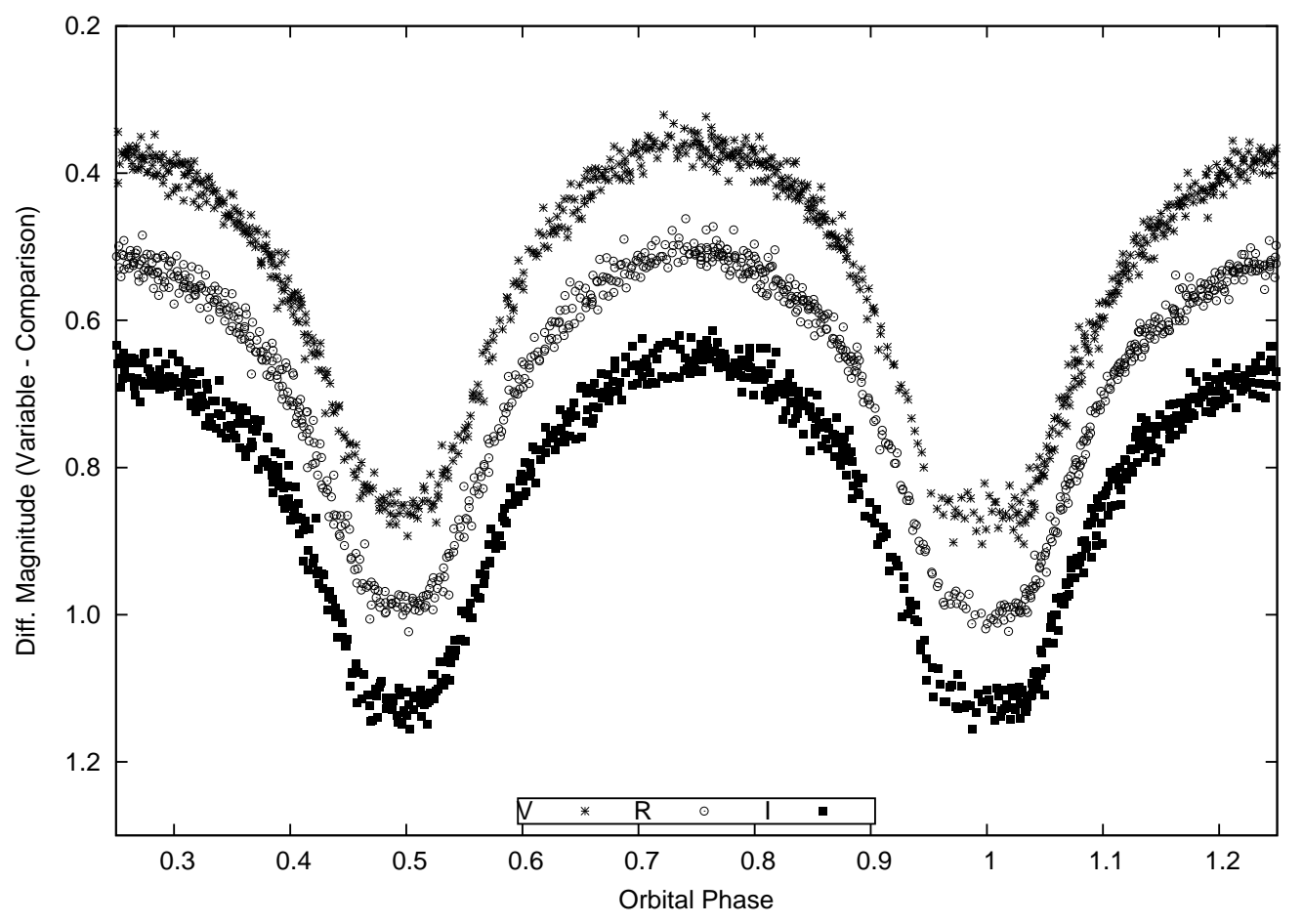

Figure 2: VRI light curves of GW Cnc obtained in 2010 and 2011 at AUO. 


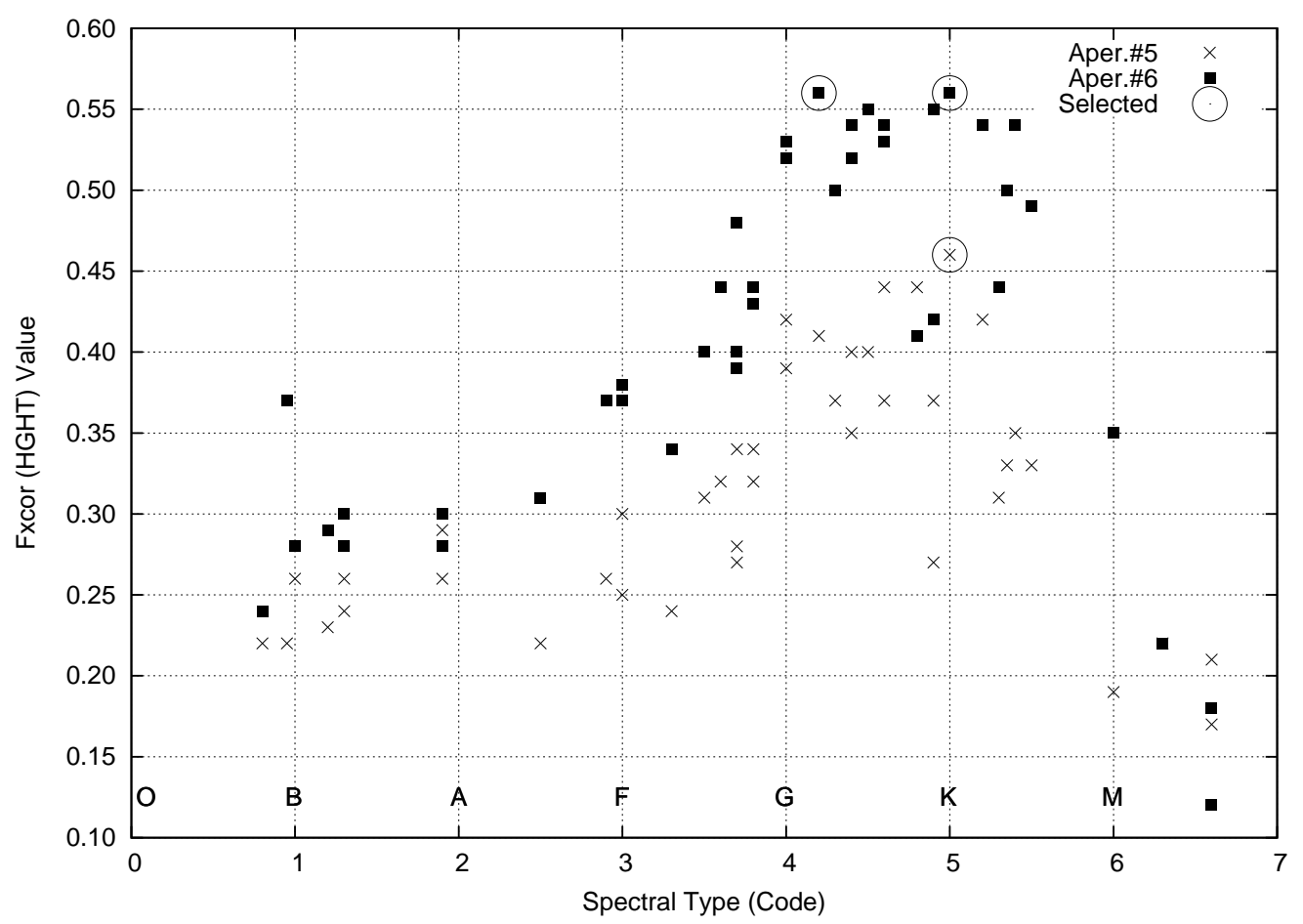

Figure 3: Comparison of GW Cnc's spectra for different spectral standards. The comparison is getting higher (HGHT) values when two spectra are matching with each other. The resulting spectrum is K0V or G2IV. 


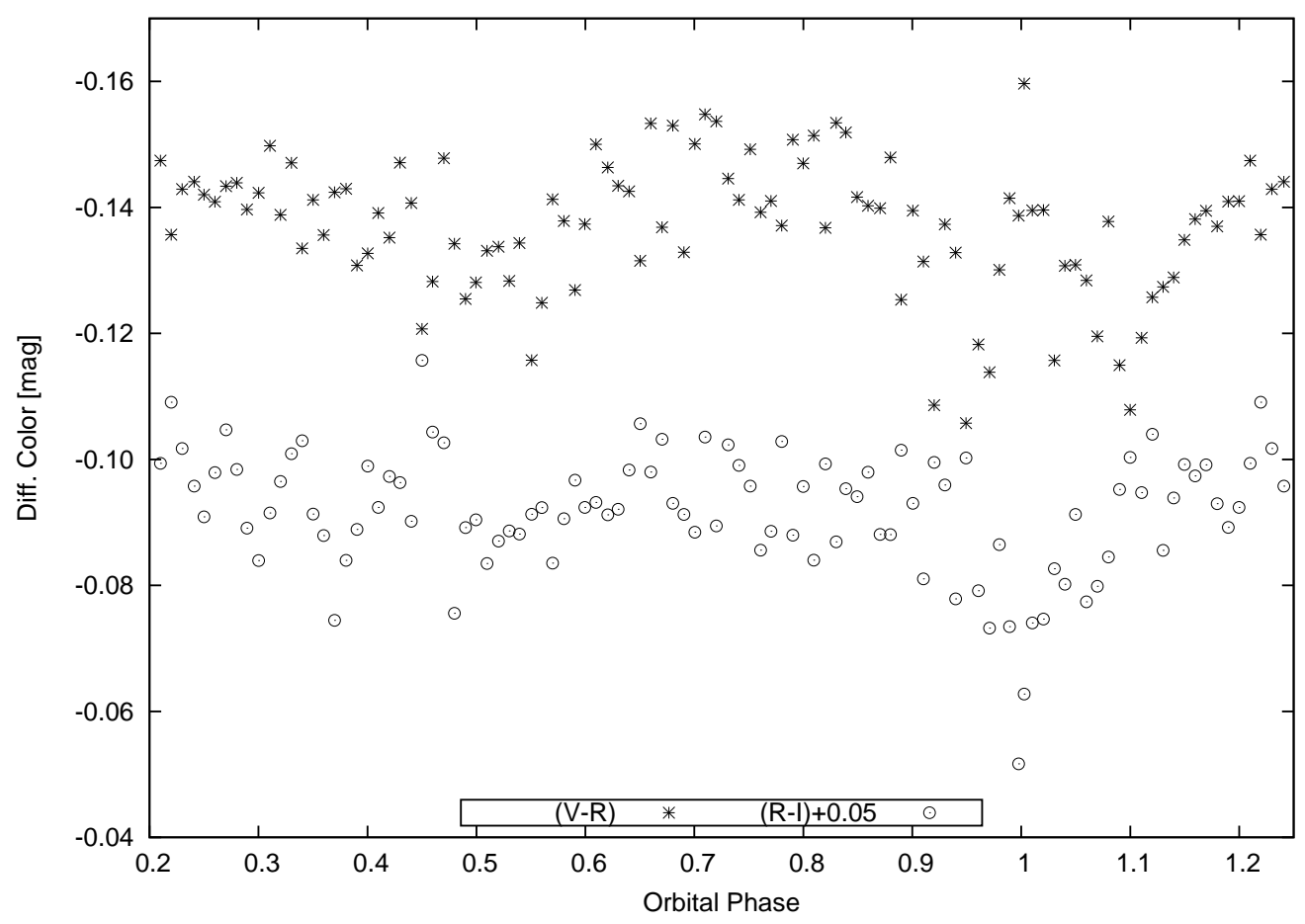

Figure 4: Differential color curves of GW Cnc. 


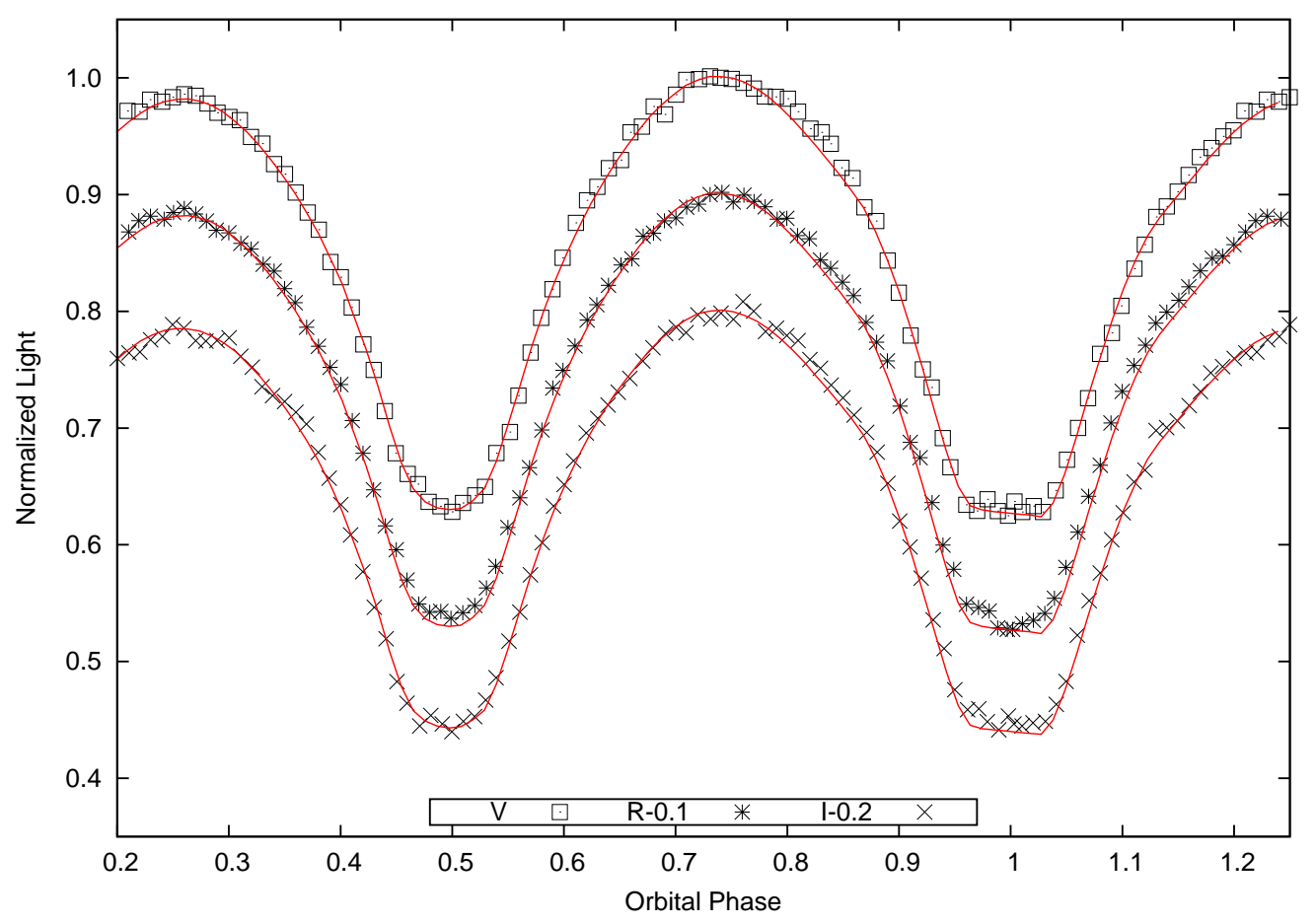

Figure 5: The observational and theoretical light curves of GW Cnc. The symbols represent the observational data for $V R I$ filters and the lines the theoretical light curves for over-contact mode. 


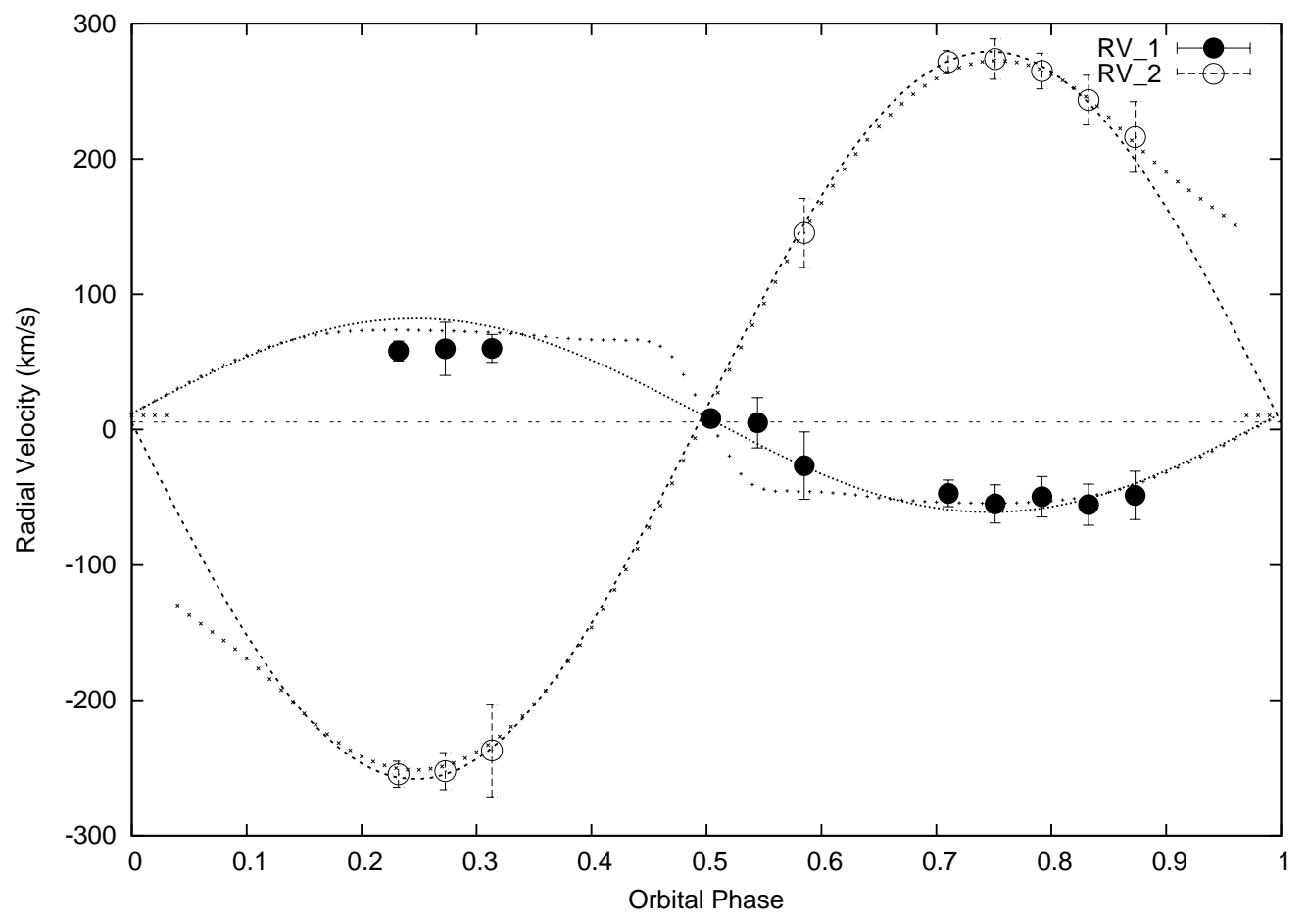

Figure 6: Radial velocity curve of GW Cnc. Solid curves are the computed radial velocity curves. 


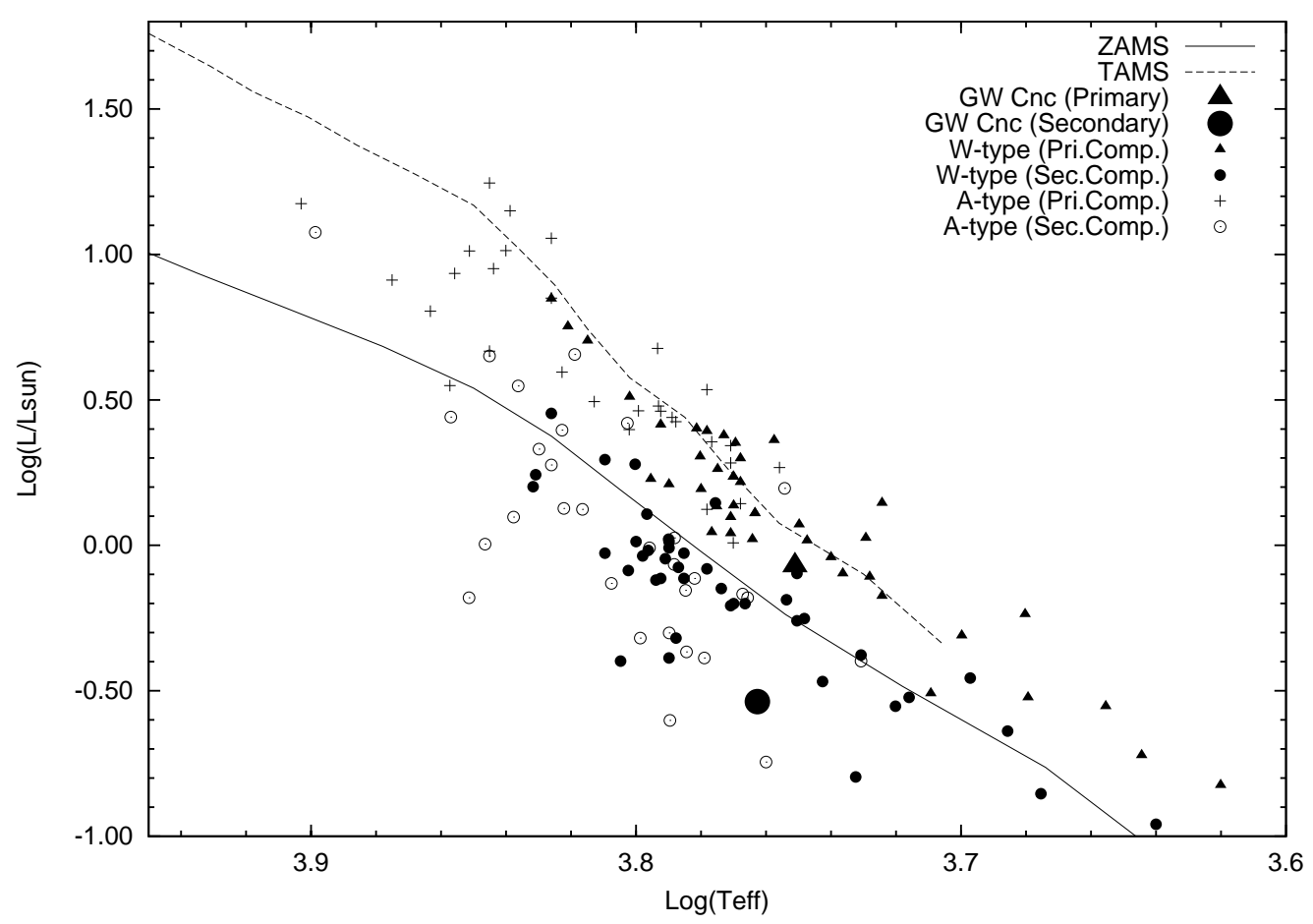

Figure 8: The location of the components of GW Cnc in the $\log T-\log L$ diagram. The sample of W- and A-type systems was obtained from Yakut \& Eggleton (2005). ZAMS (lower) and TAMS (upper) lines for solar-metallicity are obtained from Girardi et al. Girardi et al. (2000). (Notice that in this diagram the names "primary" and "secondary" have been reversed from the ones used throughout this paper, for reasons explained in the text). 
Table 1: Observation Log and standard errors of the observations of GW Cnc.

\begin{tabular}{cccccl} 
Obs.Date & $\begin{array}{c}\text { Number of Obs. } \\
(\mathrm{V}+\mathrm{R}+\mathrm{I})\end{array}$ & $\pm \sigma(V)$ & $\pm \sigma(R)$ & $\pm \sigma(I)$ & \\
\hline $2010-10-30$ & 56 & 0.013 & 0.016 & 0.015 & YD-TO \\
$2010-11-01$ & 315 & 0.013 & 0.010 & 0.015 & AO-SC \\
$2010-11-02$ & 270 & 0.017 & 0.012 & 0.015 & GG-YK \\
$2010-11-07$ & 438 & 0.016 & 0.012 & 0.015 & ZT-AB \\
$2010-11-10$ & 353 & 0.018 & 0.014 & 0.020 & GG-HA \\
$2010-11-14$ & 393 & 0.017 & 0.020 & 0.025 & HG-GV \\
$2010-11-18$ & 85 & 0.011 & 0.012 & 0.011 & HG \\
$2011-03-14$ & 291 & 0.015 & 0.010 & 0.011 & AO-YS \\
$2011-03-15$ & 292 & 0.017 & 0.016 & 0.016 & GG-YK \\
\hline & Mean $=$ & 0.015 & 0.014 & 0.016 &
\end{tabular}

Observers: YD: Y. Demircan, TO: T. Ozun, AO: A. Okan, SC: S. Cerit, GG: G. Gökay, YK: Y. Kılıç, ZT: Z. Terzioğlu, AB: A. Baykal, HA: H. Arslan, HG: H. Gürsoytrak, GV: G. Varol, YS: Y. Şendă̆.

Table 2: Magnitudes and color values of the variable and comparison stars from "All-sky spectrally matched Tycho2 stars" by Pickles \& Depagne (2010).

\begin{tabular}{rccccccccc}
\hline Star & B & V & R & I & J & H & K & B-V & Sp.Type \\
\hline GW Cnc & 13.47 & 12.716 & 12.28 & 11.89 & 11.35 & 11.04 & 10.96 & 0.754 & G8V \\
TYC1399-1572-1 & 12.96 & 11.629 & 10.96 & 10.43 & 9.31 & 8.68 & 8.51 & 1.331 & rK3III \\
GSC1399-1348 & 13.02 & 12.501 & 12.19 & 11.90 & 11.51 & 11.25 & 11.20 & 0.519 & wF8 \\
GSC1399-2073 & 13.41 & 12.841 & 12.49 & 12.15 & 11.76 & 11.49 & 11.43 & 0.569 & rF8V \\
\hline
\end{tabular}


Table 3: Times of minimum light and $(\mathrm{O}-\mathrm{C})$ residuals of GW Cnc calculated by using the light elements given by Khruslov (2005).

\begin{tabular}{cccllcl}
\hline HJD+2400000 & $\pm \sigma$ & Ecl.Type & Method & E & O-C & Ref. \\
\hline 51554.0230 & - & p & Survey & 0 & 0.00000 & 1 \\
54172.2949 & 0.0044 & p & pe -Ir & 9304 & -0.01326 & 2 \\
54172.4346 & 0.0019 & s & pe -Ir & 9304.5 & -0.01427 & 2 \\
54172.5766 & 0.0019 & p & pe -Ir & 9305 & -0.01297 & 2 \\
54506.3336 & 0.0006 & p & pe -Ir & 10491 & -0.01417 & 3 \\
54523.4952 & 0.0004 & p & ccd c & 10552 & -0.01893 & 4 \\
54831.9258 & 0.0004 & p & ccd & 11648 & -0.01912 & 5 \\
54869.0690 & - & p & pe V & 11780 & -0.02270 & 6 \\
55502.5278 & 0.0001 & p & ccd BVRI & 14031 & -0.02906 & 7 \\
55508.5771 & 0.0001 & s & ccd BVRI & 14052.5 & -0.03019 & 7 \\
55515.6124 & 0.0002 & s & ccd BVRI & 14077.5 & -0.03026 & 7 \\
55571.8950 & 0.0002 & s & pe V & 14277.5 & -0.03066 & 8 \\
55635.3537 & 0.0003 & p & ccd BVRI & 14503 & -0.03105 & 7 \\
55636.3383 & 0.0007 & s & ccd BVRI & 14506.5 & -0.03140 & 7 \\
55656.7408 & 0.0002 & p & pe V & 14579 & -0.03148 & 8 \\
55932.9471 & 0.0005 & s & ccd & 15560.5 & -0.03401 & 9 \\
56007.3788 & 0.0002 & p & ccd R & 15825 & -0.03658 & 10 \\
56007.3793 & 0.0001 & p & ccd I & 15825 & -0.03608 & 10 \\
56007.3796 & 0.0002 & p & ccd V & 15825 & -0.03578 & 10 \\
56021.7252 & 0.0012 & p & ccd V & 15876 & -0.04234 & 11 \\
56359.7073 & 0.0004 & p & ccd c & 17077 & -0.03966 & 12 \\
\hline
\end{tabular}

References: 1. Khruslov (2005), 2. Hübscher (2007), 3. Hübscher et al. (2010), 4. Brat et al. (2008), 5. Diethelm (2009), 6. Kazuo (2010), 7. Gökay et al. (2012), 8. Diethelm (2011), 9. Diethelm (2012a), 10. Honkava et al. (2013), 11. Diethelm (2012b), 12. Nelson (2014). 
Table 4: Radial velocities of the primary and secondary components obtained by using CCF method located in the IRAF RV task. $\sigma$ is the standard deviation of the calculated radial velocities obtained for the suitable orders.

\begin{tabular}{|cc|rl|rl|}
\hline $\begin{array}{c}\text { HJD } \\
2400000+\end{array}$ & Phase & \multicolumn{2}{|c|}{$\begin{array}{c}\text { (km/s) } \\
(\mathrm{km} / \sigma\end{array}$} & $\begin{array}{c}R V_{2} \\
(\mathrm{~km} / \mathrm{s})\end{array}$ \\
\hline 55521.5236 & 0.504 & 8.15 & 5.05 & - & - \\
55521.5350 & 0.544 & 5.09 & 18.60 & - & - \\
55521.5465 & 0.585 & -26.59 & 24.85 & 145.31 & 25.54 \\
55521.5818 & 0.711 & -47.13 & 9.86 & 271.59 & 8.51 \\
55521.5932 & 0.751 & -54.91 & 14.07 & 273.83 & 14.97 \\
55521.6047 & 0.792 & -49.57 & 14.95 & 265.03 & 13.11 \\
55521.6161 & 0.832 & -55.39 & 15.21 & 243.53 & 18.32 \\
55521.6275 & 0.873 & -48.58 & 17.81 & 216.19 & 26.10 \\
55522.5728 & 0.232 & 58.13 & 7.32 & -254.73 & 9.62 \\
55522.5842 & 0.273 & 59.67 & 19.61 & -252.36 & 13.68 \\
55522.5956 & 0.313 & 59.99 & 10.27 & -237.09 & 34.24 \\
\hline
\end{tabular}


Table 5: The light and radial velocity curve solutions of GW Cnc. Assumed parameters are marked with asterix, ${ }^{*}$.

\begin{tabular}{|c|c|c|}
\hline Parameters & VRI & $\pm \sigma$ \\
\hline $\mathrm{a}\left(\mathrm{R}_{\odot}\right)=$ & 1.936 & 0.010 \\
\hline$V_{\gamma}(\mathrm{km} / \mathrm{s})=$ & 8.71 & 1.2 \\
\hline$K_{1}(\mathrm{~km} / \mathrm{s})=$ & 274.97 & - \\
\hline$K_{2}(\mathrm{~km} / \mathrm{s})=$ & 72.85 & - \\
\hline$i\left(^{\circ}\right)=$ & 83.38 & 0.25 \\
\hline$T_{1}(K)=$ & $5790^{*}$ & 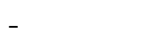 \\
\hline$T_{2}(K)=$ & 5649 & 6 \\
\hline$q=m_{2} / m_{1}=$ & 3.7729 & 0.0070 \\
\hline$A_{1}=A_{2}=$ & $0.50^{*}$ & - \\
\hline$g_{1}=g_{2}=$ & $0.32^{*}$ & - \\
\hline$L_{1} / L_{\text {Tot. }}(\mathrm{V})=$ & 0.24505 & 0.00093 \\
\hline$(R)=$ & 0.24112 & 0.00073 \\
\hline$(I)=$ & 0.23799 & 0.00060 \\
\hline$L_{2} / L_{\text {Tot. }}(\mathrm{V})=$ & 0.75506 & 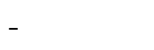 \\
\hline$(R)=$ & 0.75900 & - \\
\hline$(\mathrm{I})=$ & 0.76214 & - \\
\hline$x_{1}($ bolo $)=$ & $0.648^{*}$ & - \\
\hline$x_{2}($ bolo $)=$ & $0.649^{*}$ & - \\
\hline$y_{1}($ bolo $)=$ & $0.207^{*}$ & - \\
\hline$y_{2}($ bolo $)=$ & $0.193^{*}$ & - \\
\hline$r_{1}($ pole $)=$ & 0.26085 & 0.00038 \\
\hline$r_{1}($ side $)=$ & 0.27271 & 0.00046 \\
\hline$r_{1}($ back $)=$ & 0.31282 & 0.00086 \\
\hline$r_{2}($ pole $)=$ & 0.47352 & 0.00056 \\
\hline$r_{2}($ side $)=$ & 0.51306 & 0.00080 \\
\hline$r_{2}($ back $)=$ & 0.54005 & 0.00105 \\
\hline$\overline{\Omega_{1}}=\Omega_{2}=$ & 7.48435 & 0.00838 \\
\hline$\Omega_{i n}=$ & 7.62186 & - \\
\hline$\Omega_{\text {out }}=$ & 6.16081 & 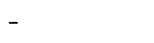 \\
\hline Fillout: $f_{1}=f_{2}=$ & $9.41 \%$ & 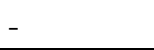 \\
\hline Spot Latitude $\left({ }^{\circ}\right)=$ & 85.66 & Secondary \\
\hline Spot Longitude $\left(^{\circ}\right)=$ & 113.26 & $\ldots$ \\
\hline Spot $\operatorname{Radius}\left({ }^{\circ}\right)=$ & 14.59 & $\ldots$ \\
\hline Spot Temp. Factor $=$ & 0.9067 & \\
\hline$\sum(O-C)^{2}=$ & 28.70 & $\left(R V_{1}\right)$ \\
\hline$=$ & 8.93 & $\left(R V_{2}\right)$ \\
\hline$=$ & 0.0119 & $(\mathrm{~V})$ \\
\hline$=$ & 0.0093 & (R) \\
\hline$=$ & 0.0127 & (I) \\
\hline
\end{tabular}


Table 6: Absolute parameters of GW Cnc.

\begin{tabular}{rll}
\hline Parameters & Primary & Secondary \\
\hline Mass $\left(\mathrm{M}_{\odot}\right)=$ & $0.257 \pm 0.004$ & $0.971 \pm 0.016$ \\
Radius $\left(\mathrm{R}_{\odot}\right)=$ & $0.526 \pm 0.003$ & $0.961 \pm 0.007$ \\
Luminosity $\left(\mathrm{L}_{\odot}\right)=$ & $0.279 \pm 0.008$ & $0.842 \pm 0.010$ \\
$M_{\text {bol }}=$ & $6.136 \pm 0.032$ & $4.936 \pm 0.017$ \\
$\log \mathrm{g}(\operatorname{cgs})=$ & $4.406 \pm 0.008$ & $4.460 \pm 0.010$ \\
$\rho(\operatorname{cgs})=$ & $2.489 \pm 0.004$ & $1.543 \pm 0.003$ \\
$\mathrm{a}\left(\mathrm{R}_{\odot}\right)=$ & $1.936 \pm 0.010$ & \\
$\mathrm{~d}(\mathrm{pc})=$ & $367 \pm 2$ & \\
\hline
\end{tabular}

